\title{
Ondřejov's double-station observation of faint meteors using a TV technique (image intensifiers Dedal): 1998-2005
}

\author{
Rostislav Štork \\ Astronomical Institute, Academy of Sciences of the Czech Republic \\ Fričova 298, CZ-251 65 Ondřejov, Czech Republic \\ email: stork@asu.cas.cz
}

\begin{abstract}
TV cameras equipped with image intensifiers are suitable and often used instruments for observing faint meteors. Our regular double-station observations with image intensifiers Dedal (type Dedal 41) started in 1998. Since 2006 we perform observations using new Mullard XX1332 intensifiers. In this paper we present an overview of the eight years of Dedal era. The amount of observing hours, number of meteors, statistics how many meteors were recognized using the automatic system MetRec or found manually, etc. are presented for each year. We also briefly overview the science results from these observations.
\end{abstract}

Keywords. Meteors; instrumentation

\section{Instrumentation}

The left panel of Fig. 1 shows image intensifiers Dedal 41 mounted in front of videocameras (S-VHS Panasonic). Dedal 41 is a second generation image intensifier with micro channel plate (MPC), which provides high gain and image quality. The positions of our stations (together with Prague) are marked on the map of the Czech Republic (right panel on Fig. 1). The distance between Ondřejov and Kunžak is $92.5 \mathrm{~km}$.

We use the objectives Arsat 1.4/50 (field of view 28 ${ }^{\circ}$ ), Flektogon 2.4/35 (field of view $40^{\circ}$ ) and Zenitar 2.8/16 (field of view $85^{\circ}$ ) for wide-field camera. The videosignal is recorded on the external S-VHS videorecorders. The time is inserted into the videoframes (Fig. 2). Originally we used a DCF inserter Cuno but now we prefer a GPS inserter AstroLab because the DCF signal is not stable enough in our area and the AstroLab digits do not cover part of the field of view as the Cuno did.

\section{Overview of observations}

Our regular double-station observation started in 1998. We usually observe major meteor showers and minor shower whenever their higher activity is expected. Since 2001 we have also been using a third camera, the wide-field one, which is located in Kunžak and covers a larger field of view. Only part of a meteor is often recorded in the field of view of normal (narrow-field) cameras. In these cases a wide-field camera allows to record the whole visible trajectory of bright meteors.

Figure 3 shows the number of observing hours per year and the number of recorded meteors. The large drop of observation in 2002 is clearly visible. This was due to very bad weather during our observing campaigns.

The number of single-station (dark column) and double-station (light column) meteors is displayed in the histogram of Fig. 3. The higher number of meteors recorded by one 

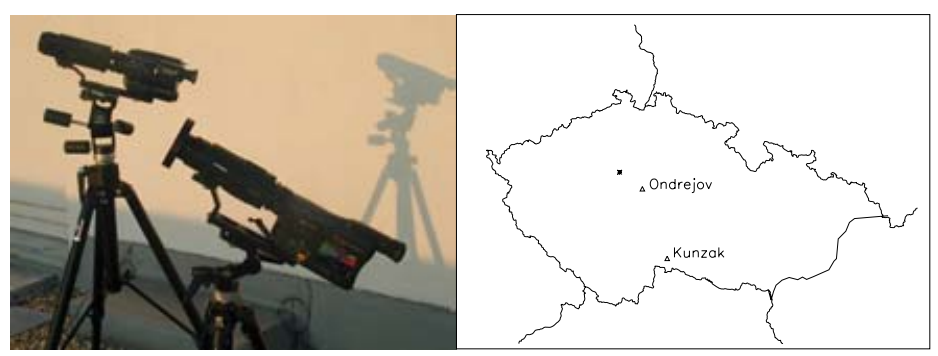

Figure 1. Left: Image intenfiers Dedal 41 mounted in front of videocameras (S-VHS Panasonic); right: the map of the Czech Republic — the positions of our stations are marked (Prague is also shown).

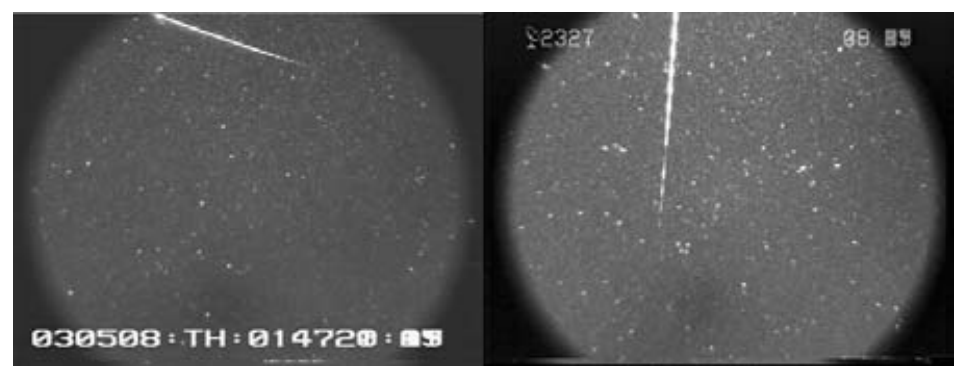

Figure 2. Examples of recorded meteors (sum images of about ten videoframes): left: with older DCF time inserter (Cuno); right: with new GPS inserter (AstroLab).
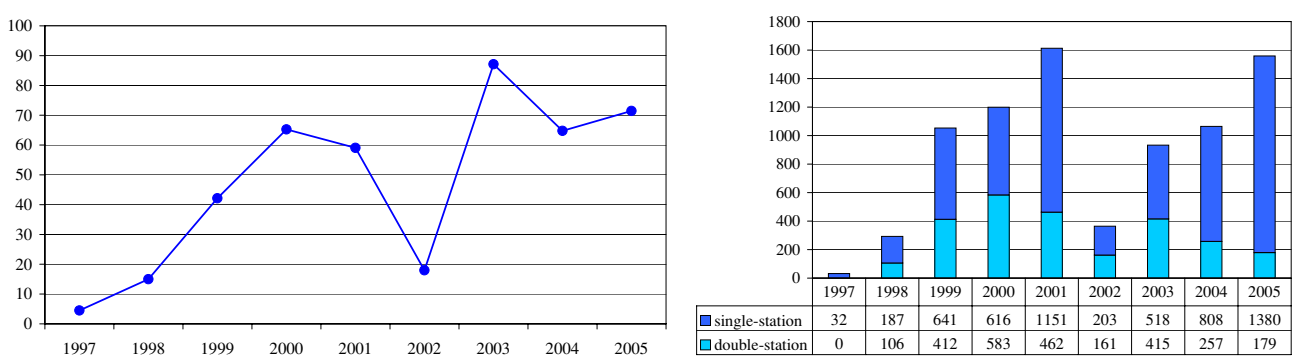

Figure 3. Observing hours per year (left) and number of recorded meteors (right).

station only is mainly caused by the use of the third camera and in 2005 also by the fact that not all the records are thoroughly processed yet.

\subsection{Meteors and individual meteor records}

We distinguish between meteors (phenomena) and meteor records (records on videotape). It means we have one record for single-station meteor and two records for double-station meteor.

The left panel on Fig. 4 shows number of meteors and individual meteor records. The total number of meteor observations is 8111 , numbers per year are shown by the solid line. The total number of individual meteor records is 11105 , numbers per year are shown by dashed line.

For every single-station meteor we check the other station videotape. The dotted line in the left graph (Fig. 4) shows how many meteors were not found on the other station record although the check was done. The number of meteors which were not found despite of the additional check of videotape is 2911 . 

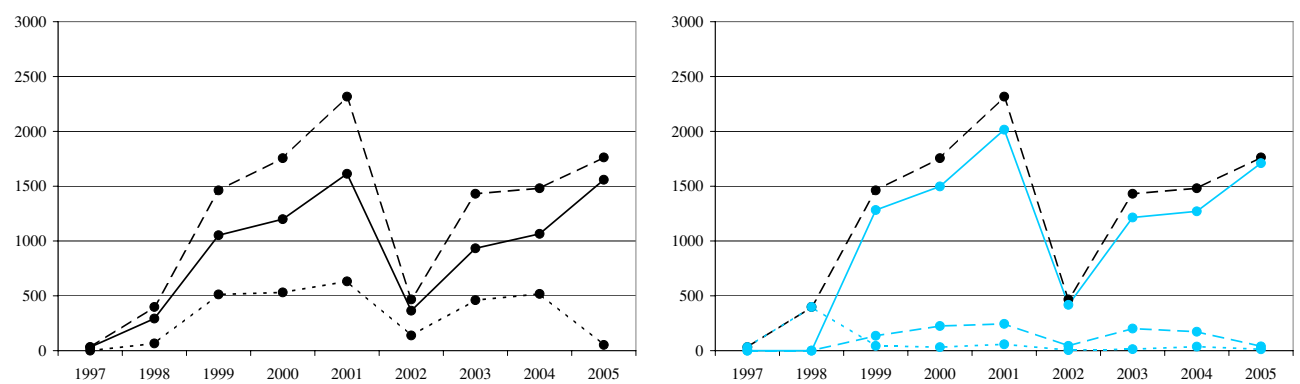

Figure 4. Left part: Number of meteors (solid) and number of individual meteor records (dashed); dotted line shows the number of meteors not found on the other station (although the tapes were additionally manually checked). Right part: the analysis of the ways we found meteors on videotapes. The majority of meteors have been found using the automatic system MetRec - solid blue (grey on b/w print) line. The dotted blue (grey) line represents number of meteors found visually (watching TV screen). The meteors found by checking a tape at the time when the meteor was detected on the other station are displayed by the dashed blue (grey) line. The dashed black line shows the total number of individual meteor records (the same as on the left graph).

The reason for not observing the meteor at one of the stations may be either worse observing conditions at that time or the fact that the meteor was outside of the field of view. We configure our cameras so that the meteors are at similar ranges from both stations and meteor apparent brightness is therefore nearly the same.

\subsection{Methods of finding meteors on videotapes}

The right panel on Fig. 4 shows the statistics of the different methods with which we used to find meteors on videotapes. Since 1999 the majority of meteors have been found using the automatic system MetRec (Molau 1998, www.metrec.org) - solid blue (grey on b/w print) line (total number 9409); the dotted blue (grey) line represents number of meteors found visually (watching TV screen) - the total number is 633. The meteors found by checking a tape at the time where meteor was detected on the second station are displayed by the dashed blue (grey) line (total number 1063). Nearly one in four checked meteors was found. The dashed black line shows the total number of individual meteor records, i.e. the sum of all (solid, dashed, and dotted) blue (grey) lines.

\section{Results}

The data from this observing program have been used in several publications and other papers are in preparation. Here we present a short list of them.

Štork et al. (2003) analysed sporadic meteors using Ceplecha's $K_{B}$ parameter (Ceplecha 1988) and found that no fragile cometary meteoroids (i.e. meteoroids with low value of $K_{B}$ ) are present on orbits with low perihelion distance.

Koten et al. (2003) presented the catalogue of the heliocentric orbits of 817 meteors recorded within our double-station program in 1998-2001. The catalogue is also available in electronic version.

Koten et al. (2004) studied light curves and atmospheric trajectories of almost 500 meteors of five major meteor showers. Although the parameter $F$ describing the shape of the light curve lays in a broad range, they succeeded to determine the typical value of this parameter for all the studied showers. Investigation of height data showed significant differences between Geminids and other showers. The Geminids proved to be the most compact meteoroids. 
Borovička et al. (2005) studied 97 spectra of mainly sporadic meteors. For the majority of them the heliocentric orbits were also known from our double-station observations. Three distinct populations of Na-free meteoroids were identified. The first population are meteoroids on asteroidal orbits containing only Fe lines in their spectra and possibly related to iron-nickel meteorites. The second population are meteoroids on orbits with small perihelia ( $q \leqslant 0.2 \mathrm{AU}$ ), where $\mathrm{Na}$ was lost by thermal desorption. The third population of Na-free meteoroids resides on Halley type cometary orbits.

Koten et al. (2006) studied 51 Quadrantid meteors. The data analysis showed that the Quadrantids are similar in some aspects to meteor showers of cometary origin, but in other aspects they are closer to the Geminid meteors. Quadrantid meteoroids have partially lost volatile component, but are not depleted to the same extent as the Geminid meteoroids. These results lead to the conclusion that the parent body is a dormant comet.

In 2005 we succeeded to observe enhanced activity of October Draconids (Koten et al., in preparation). For the first time we detected also the atmospheric deceleration of faint meteors, which can be used to describe meteoroid structure and fragmentation (Borovička et al., in preparation).

\section{Conclusions}

This paper presents a summary of data collected using Dedal image intensifiers during 1998-2005. The recorded meteors have been catalogued, digitized, analyzed and meteor trajectories are being computed.

The era of Dedal lasted for eight years and is over. We are now using new Mullard XX1332 image intensifiers. It has been tested at one station already in autumn 2005 . This intensifier has similar sensitivity but lower level of noise. The result is that the stellar limiting magnitude is about 8 for a field of view $55^{\circ}$, i.e. the same value as for Dedal with field of view $28^{\circ}$. The number of recorded meteors is several times higher.

\section{Acknowledgements}

The author would like to thank to the team members Pavel Koten, Pavel Spurný and Jiří Borovička, who participate in the observations, computation of meteors trajectories and data analysis. I am also indebted for their valuable comments in preparation of this paper. Last but not least many thanks to the referee Tadeusz Jopek and the editor David Vokrouhlický for their suggestions. This work was supported by the Academy of Sciences of the Czech Republic scientific project AV0Z10030501.

\section{References}

Borovička, J., Koten, P., Spurný, P., Boček, J. \& Štork R. 2005, Icarus 174, 15

Ceplecha, Z. 1988, Bull. Astron. Inst. Czechosl. 39, 221

Koten, P., Spurný, P., Borovička, J. \& Štork, R. 2003, Publ. Astron. Inst. ASCR, No. 91, 1

Koten, P., Borovička, J., Spurný, P., Betlem, H. \& Evans, S. 2004, A\& A 428, 683

Koten, P., Borovička, J., Spurný, P., Evans, S., Štork, R. \& Elliot, A. 2006, MNRAS 366, 1367

Molau, S. 1998, in: R. Arlt \& A. Knoefel (eds.), Proceedings of the International Meteor Conference, Stará Lesná 20-23 August 1998, p. 9

Štork, R., Koten, P., Borovička, J. \& Spurný, P. 2003, in: B. Warmbein (ed.), Proceedings of the Asteroids, Comets, Meteors (ACM 2002) conference, ESA SP-500, p. 189 